\title{
Antiproliferative Effect of Electric Fields on Breast Tumor Cells In Vitro and In Vivo
}

\author{
Firman Alamsyah $^{1 *}$, Izzatun Niswah Ajrina ${ }^{2}$, Fitriya Nur Annisa Dewi ${ }^{3,4}$, Diah \\ Iskandriati $^{3,4}$, Silvia Arin Prabandari ${ }^{4}$, Warsito P. Taruno ${ }^{1}$ \\ ${ }^{1}$ Center for Medical Physics and Cancer Research Ctech Labs Edwar Teknologi, Tangerang, Indonesia \\ ${ }^{2}$ Dept. of Biology Faculty of Science and Technology Airlangga University, Malang, Indonesia \\ ${ }^{3}$ Bimana Indomedical, Bogor, Indonesia \\ ${ }^{4}$ Primate Research Center Bogor Agricultural University, Bogor, Indonesia
}

\begin{abstract}
Our research focused on the antiproliferative effect of low intensity (I8 VPP) and intermediate frequency $(100 \mathrm{KHz})$ electrostatic wave between two capacitive electrodes on breast tumor cells in vitro and in vivo. In vitro study has been conducted by using MCF-7 cell lines treated with external electrostatic for 24,48 , and 72 hours of treatment and the cells number were calculated during treatment by using hemocytometer and presented as Growth Inhibition (GI)\% efficacy. For in vivo, we used female mice (Mus musculus) strain $\mathrm{C} 3 \mathrm{H}$ as animal model. The mice were injected with either MCF-7 cells, mammary tumor cells from $\mathrm{C} 3 \mathrm{H}$ donor, or $\mathrm{NaCl} 0.9 \%$ (placebo) subcutaneously into the axilla area and exposed by external electrostatic in each cage for 12 hours in 2 weeks before necropsied. The adjacent and breast tissue were collected and stained with Hematoxylin-Eosin then analyzed for histopathological profile. In vitro study revealed the number of exposed cells decreased with lower proliferation rate than the non-exposed cells. Moreover, the external electrostatic caused 28-39\% growth inhibition efficacy of MCF-7 cells. After 2 weeks of exposure, placebo mice were physically normal, whereas the tumor undergone significant shrinkage of more than $67 \%$ in size. Histopathological analysis of the mammary glands indicated infiltration of macrophages into the tumor area through the blood vessel. No abnormality was found in the skin layer and mammary glands of the breast tissue of placebo mice. Here, we present new knowledge of electro-capacitive cancer therapy (ECCT) as a novel treatment modality.
\end{abstract}

Keywords : ECCT, tumor, in vitro, in vivo, breast cancer cells, antiproliferative

\section{INTRODUCTION}

Living cells exert electric fields associated with cell function (Qiao, et al., 2010). The intensity of the electric fields within a cell is less than $10 \mathrm{~V} / \mathrm{cm}$ (Kirson, et al., 2007), but within cell membrane they may reach $10^{5} \mathrm{~V} / \mathrm{cm}$, and cancer cells possess higher cellular potential than normal cells (Binggeli and Cameron, 1980). Homogenous electric fields orient polar molecules along their force lines (Zhao, et al., 1999), whereas inhomogenous electric fields direct polar molecules toward higher field intensity, demonstrating dielectrophoresis (Ma, et al., 2011; Tsutsui and Ho, 2009). Electric fields of alternating currents (ac) force polar molecules to oscillate and their effect is reduced when their frequency is elevated, except for heating (Kirson, et al., 2007). Therefore, ac fields of $100 \mathrm{kHz}$ or above are known to have no significant biological effects (Kirson, et al., 2004), although some nonsignificant effects have been reported (Takashima and Schwan, 1985; Dalton, et al., 2004; Mahaworasilpa, et al., 1996; Zimmermann, et al., 1981).

The force exerted by electric fields on polar molecules is maximal when the dipole is oriented in the direction of the fields (Bethlem, 2002). In this regard, cancer cells during mitosis contain highly polar, spatially oriented microtubules (Pasquier and Kavallaris, 2007) and that they would be affected with maximal force when the mitotic process oriented along the lines of force of the fields (Kirson, et al., 2004).

*Corresponding author e-mail: firman.alamsyah@ctechlabs.com 
The spatially oriented microtubules consist of $\alpha$ and $\beta$ tubulin heterodimers that have large electric dipole moments (Pasquier and Kavallaris, 2007), thus could be disoriented by the forces of the electric fields (Dujovne, et al., 2008).

The purpose of this study is to investigate the anti-proliferative effects of electric fields on tumor cells in cultures and animal model. We demonstrate here that electric fields, termed electro-capacitive cancer therapy (ECCT), is effective in cell culture settings, with a similar potential in animal model. These promising results raise the possibility that ECCT could become a novel treatment modality for cancer.

\section{MATERIALS AND METHODS}

\section{Cell Culture}

In vitro study was conducted at Institute of Tropical Disease Airlangga University. MCF-7 cell cultures were grown in $\alpha \mathrm{MEM}$ plus $20 \%$ FBS, $1 \%$ penicillin-streptomycin, and $1 \%$ fungizone in a $\mathrm{CO}_{2}$ incubator $\left(5 \% \mathrm{CO}_{2}\right)$ at $37^{\circ} \mathrm{C}$. Cell suspension $\left(40 \mu \mathrm{L} ; 1 \times 10^{4}\right.$ cells $\left./ \mathrm{mL}\right)$ were placed in 24-wells microplates loaded with $960 \mu \mathrm{L}$ medium (total $1000 \mu \mathrm{L} /$ well). A pair of capacitive electrodes positioned flanking on top and bottom of the microplate was connected to a square function oscillator (Fig. 1). One-directional field was generated between the pair of capacitive electrodes and alternate every $0.5 \mathrm{~ms}$.
Cell cultures were treated with external electrostatic wave and incubated for 24,48 , and 72 hours with 4 replications each. A control group with 4 replications was also incubated at the same time. At the end of the treatments, the cell number was measured using microscope counting chamber (hemocytometer). The cell number was calculated as growth inhibition (GI) efficacy of MCF-7 cells using the formula:

$\% \mathrm{GI}=\frac{\text { control cell number }- \text { treatment cell number } \times 100 \%}{\text { control cell number }}$

The data was analyzed using one way ANOVA followed by Duncan test to find statistical significance.

\section{Animal Model}

A pilot trial of the safety and efficacy of ECCT was performed in $9 \mathrm{C} 3 \mathrm{H}$ mice (Mus musculus). All procedures involving animals were performed following approval by the Institutional Care and Use Committee PT Bimana Indomedical. Animals were divided into three groups (each consisted of 3 mice), whereby each group recieved either $\mathrm{MCF}-7$ cells (MCF-7 group), adenocarcinoma cells from $\mathrm{C} 3 \mathrm{H}$ donor (tumor group), or $\mathrm{NaCl} 0.9 \%$ (placebo group) by subcutaneous inoculation into the axilla area. Following the development of palpable mass in the mammary area, animals in the tumor and placebo groups were exposed to external electrostatic wave individually in each of their cages for 12 hours per day for 2 weeks (Fig. 2).

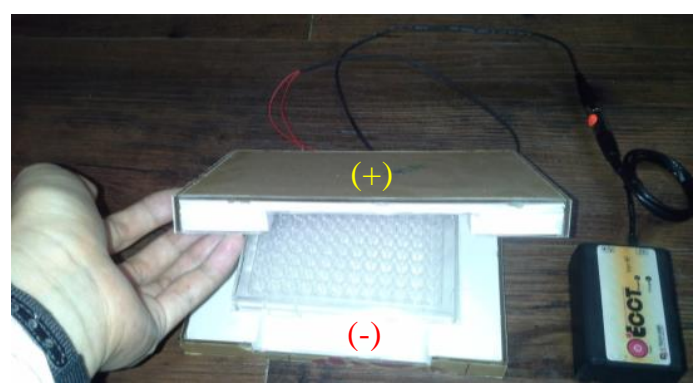

Figure I. A microplate flanked between two capacitive electrodes. The ECCT oscillator with low intensity (I8 VPP) generates electric wave to the capacitive electrodes through the wires. 
(A)

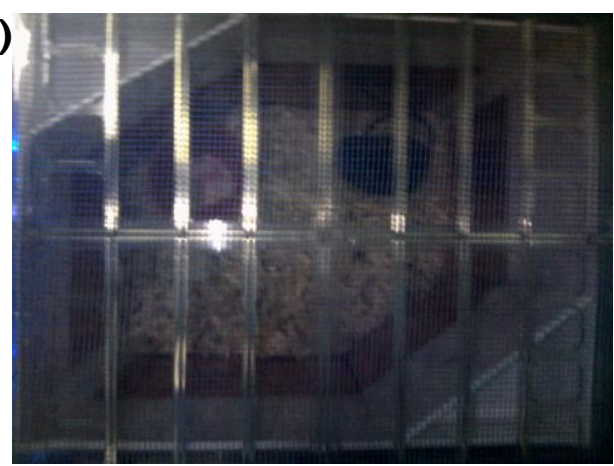

(B)

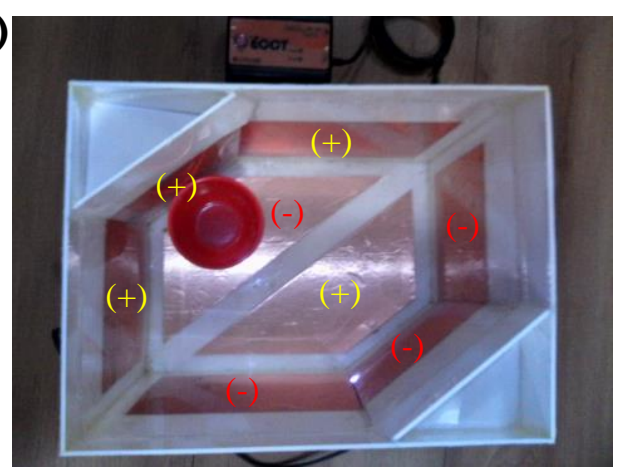

Figure 2. Electro-capacitive cancer therapy cage. (A) a treated mice inside, (B) empty cage. The electrodes are attached to the acrylic wall of the cage with opposite polarity facing each other resulting multiple field directions.

All animals were assessed 3 times a week for body weight and tumor size. Tumor size was calculated by multiplying maximal tumor length by maximal tumor width using a caliper. After 2 weeks of exposure, the mice were anesthetized using intraperitoneal injection of ketamine $(90-120 \mathrm{mg} / \mathrm{kg}$ ) and xylazine $(10 \mathrm{mg} / \mathrm{kg})$ for blood collection.

\section{RESULTS AND DISCUSSIONS}

\section{MCF-7 Cells Proliferation Rate Under} the Exposure of External Electric Field

The effects of 24, 48, and 72 hours external electric field exposure on MCF-7 cells proliferation are illustrated in Fig. 3. It describes the number of unexposed (control) cells increase with higher proliferation rate than the exposed cells. In vitro study revealed 28 $39 \%$ growth inhibition efficacy of MCF-7 cells (Fig. 4) with statistical significance using one way ANOVA $(p<0.05)$ followed by Duncan test $(\alpha=0.05$, confidence level $=95 \%)$. Based on
Further, the anesthetized mice were euthanized by cervical dislocation for breast tissue collection. The tumors and their adjacent tissues in the area of mammary glands were fixed, stained (Hematoxylin-Eosin) and analyzed histopathologically. No statistical hypothesis testing was conducted because of the small sample size. This study will be continued with bigger sample size. Duncan test result, 24 hours exposure to external electric field at $100 \mathrm{kHz}$ caused the highest significant inhibition of MCF-7 cells proliferation. Previously, Mursilatun (2010) had checked whether normal fibroblast vero cell cultures were affected by $100 \mathrm{kHz}$ external electric field. Slightly no difference in cell number between control and electric fieldtreated fibroblast vero cell cultures was observed (data not shown). Therefore, the electro-capacitive cancer therapy is proven not cause harmful effects on normal fibroblast vero cells.

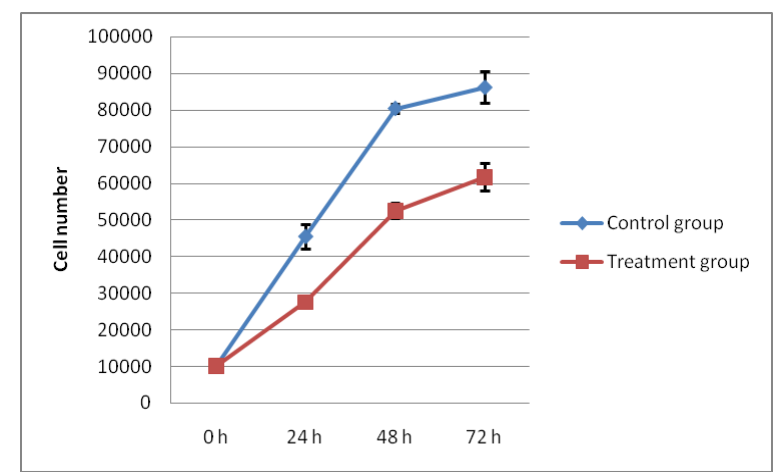

Figure 3. The effect of external electric field on proliferation of exposed (treatment) and unexposed (control) MCF-7 cells. ECCT inhibited the proliferation of MCF-7 cells in the treatment group. 


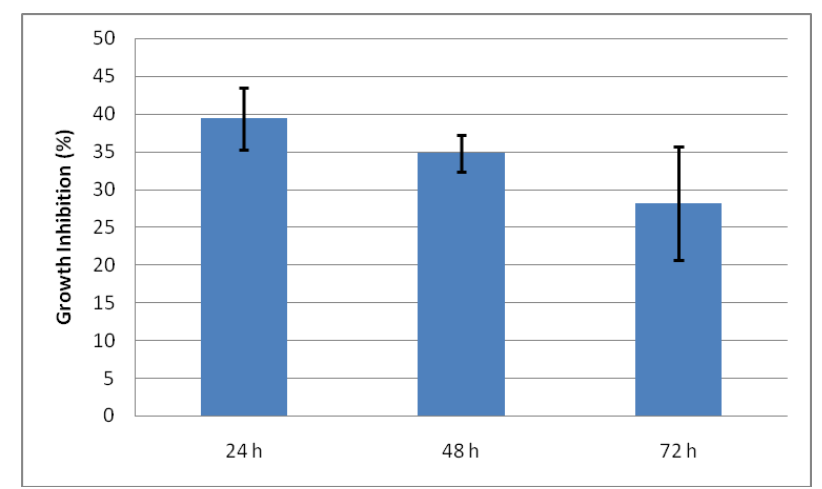

Figure 4. Growth inhibition efficacy of MCF-7 cells under external electric field exposure. The 24 hours of exposure of ECCT caused the highest significant inhibition of MCF-7 cells proliferation with statistical significance.

Two main processes occur at the cellular level during exposure to external electric field: arrest of proliferation and cell destruction. In treated cells under external electric field exposure, mitosis was began normally but at the end was extended to some periods of time (average within $2 \mathrm{~h}$ ) before cytokinesis (Kirson, et al., 2004). Furthermore, exposed cancer cells were destroyed as the formation of the cleavage furrow approached cytokinesis. During this process, the cell membrane break down and many small membrane blebs formed, resembling post-mitotic apoptotic cell death (Shinomiya, 2001). This cancer cell destruction was dependent on the orientation of the division process in relation to the field vectors. At the subcellular level, external electric field disrupt the normal polymerization-depolymerization process of microtubules during mitosis (Kirson, et al., 2004). Cancer cell destruction has been demonstrated under the exposure of external electric field of ECCT (Sabrina, 2014).

\section{Breast tumor proliferation rate in the animal model under the exposure of external electric field.}

To test whether electro-capacitive cancer therapy is effective to inhibit the proliferation of breast tumor cells in vivo, we tested their effect on $\mathrm{C} 3 \mathrm{H}$ mice (Mus musculus) animal model. After inoculation of MCF-7 tumor cells, none of the animals developed tumor, whereas inoculation of adenocarcinoma cells from $\mathrm{C} 3 \mathrm{H}$ donor resulted in development of palpable mass in the breast of recipient animals, up to the size of 8.59-10.91 mm in length. After 2 weeks of exposure to external electric fields, placebo mice were physically normal, whereas animals that initially showed palpable mass underwent a significant shrinkage of the tumor, more than $67 \%$ in size. The results suggest that $100 \mathrm{kHz}$ of external electric fields at low intensity may inhibit the proliferation of breast tumor cells.

During in vivo experiment, no significant changes were observed in the complete blood count of the tumor-implanted and placebo mice (Fig. 5). Histopathological analysis of mammary glands revealed adenoma tubular type formed by epithelial cells. This adenoma would be tumor remnants, contiguous with the invasive cancer, adenocarcinoma (Leslie, et al., 2002). Furthermore, macrophages and other immune cells infiltrate through the blood vessel in the area of breast tumor (Fig. 6). No abnormality was found in the skin layer and mammary gland of placebo mice (Fig. 7). The findings may be consistent with how macrophages would digest any debris resulted from tumor cell destruction (Brooker, et al., 2008) and also participate in the clearing of apoptotic cells (Teo, 2003). In addition, macrophages are a major cell component infiltrating certain tumors, such as breast cancer (Weigert, et al., 2007). Importantly, electrocapacitive cancer therapy performed in this study was well tolerated by the animals as seen by the absence of clinical abnormality.

The anti-proliferative effect of external electric fields has been attributed to two separate mechanisms as mentioned above: interference with the formation of the mitotic spindle microtubules and physical destruction of cells during cleavage, both of which are strongly dependent on the orientation of mitosis axis versus the field vectors (Kirson, et al, 2004). Because the relative orientation of the mitosis axis during cytokinesis is random, it would be expected that only a fraction of 
dividing cells would be affected by extenal electric field generated by the electro-capacitive cancer therapy of any specific direction. To overcome this problem, we applied several field directions on the in vivo experiment resulting from the positions of the capacitive electrode pairs (Fig. 2b) and have shown that increasing the number of field directions, resulted in potentially anti-proliferative effect of electro-capacitive cancer therapy in vivo (>67\%) as compared to in vitro $(28-39 \%)$.

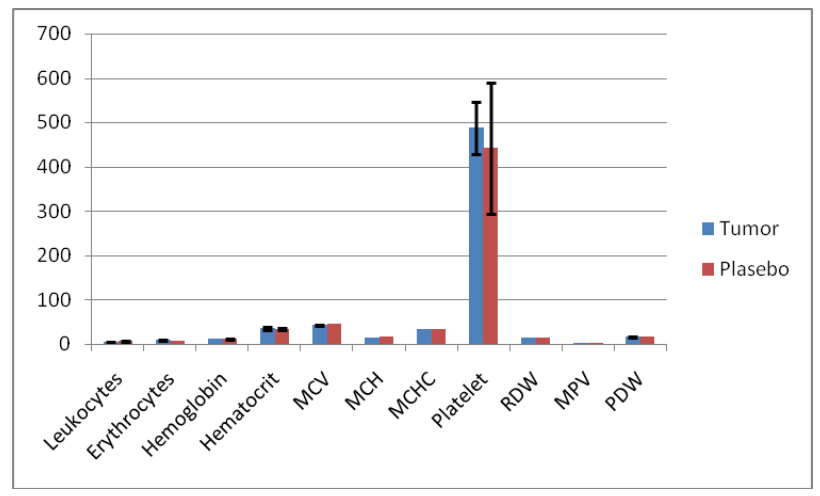

Figure 5. Complete blood count of the tumor-implanted and placebo mice. The unit of each parameter are as follow: leukocytes $(103 / \mu \mathrm{L})$, erythrocytes $(106 / \mu \mathrm{L})$, hemoglobin $(\mathrm{g} / \mathrm{dL})$, hematocrit $(\%)$, MCV/Mean Corpuscular Volume (fL), MCH/Mean Corpuscular Hemoglobin (pg), MCHC/Mean

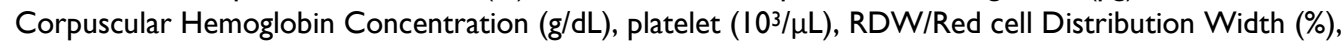
MPV/Mean Platelet Volume (fL), PDW/Platelet Distribution Width (\%).

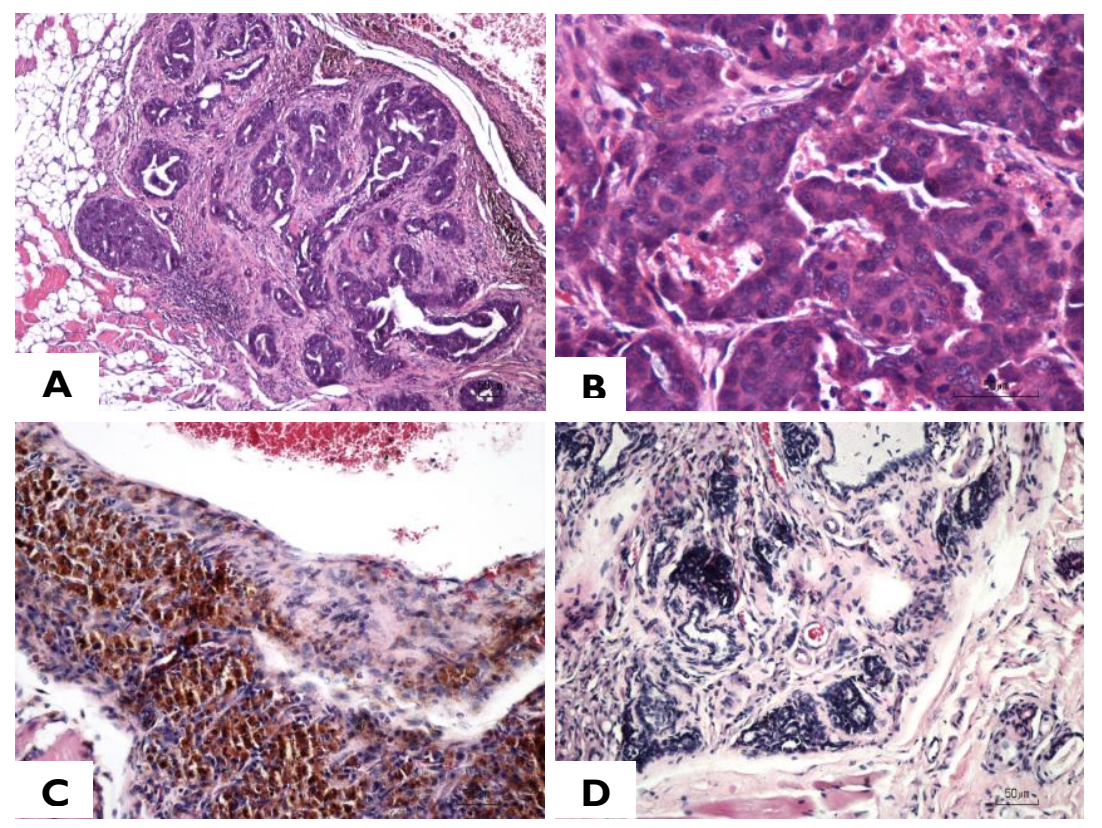

Figure 6. (A) Adenoma, tubular type. (B) Tubules formed by epithelial cells. (C) Extensive infiltration of macrophage with hemosiderin accumulation on the wall of the blood vessel. (D) The infiltration of lymphocytes around the blood vessels. 


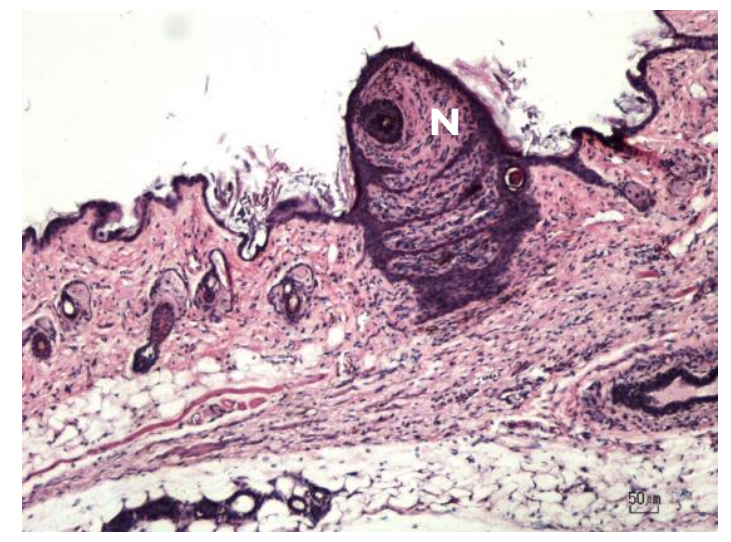

Figure 7. The skin layer and mammary gland of placebo mice; $\mathbf{N}$, nipple

In summary, we demonstrated that electro-capacitive cancer therapy is effective in arresting the proliferation of breast tumor cells in culture settings, with a similar potential in solid breast tumors in animal model. Importantly, no general side effect or local histopathological damage was observed.

\section{CONCLUSION}

The highly specific effects of ECCT on dividing cells, together of the relative ease of applying this treatment, make it an attractive candidate to serve as a novel treatment modality in cancer.

\section{REFERENCES}

Bethlem, H.L., 2002, Deceleration and Trapping of Polar Molecules Using Time-varying Electric Fields, Thesis, Katholieke Universiteit Nijmegen, Netherlands.

Binggeli, R. and Cameron, I.L., 1980, Cellular Potentials of Normal and Cancerous Fibroblasts and Hepatocytes, Cancer Res., 40, I830-1835.

Brooker, R.J., Widmaier, E.P., Graham, L.E. and Stiling, P.D., 2008, Biology, New York: McGraw Hill.

Dalton, C., Goater, A. D., Burt, J.P. and Smith, H. V., 2004, Analysis of Parasites by Electrorotation, J. Appl. Microbiol., 96(I), 24-32.

Dujovne, I., van den Heuvel, M., Shen, Y., de Graaff, M. and Dekker, C., 2008, Velocity Modulation of Microtubules in Electric Fields, Nano Lett., 8(I2), 42I720.
Kirson, E.D., Gurvich, Z. Schneiderman, R.D., Itzhaki, A., Wasserman, Y., et al., 2004, Disruption of Cancer Cell Replication by Alternating Electric Fields, Cancer Res., 64(9), 3288-3295.

Kirson, E.D., Dbaly, V., Tovarys, F., Vymazal, J., Soustiel, J. F., Itzhaki, A., et al., 2007, Alternating Electric Fields Arrest Cell Proliferation in Animal Tumor Models and Human Brain Tumors, Proc. Natl. Acad. Sci. USA, 104(24), I0I52-I0I57.

Kirson, E.D., Schneiderman, R.S., Dbaly, V., Tovarys, F., Vymazal, J., Itzhaki, A., et al., 2009, Chemotherapeutic Treatment Efficacy and Sensitivity are Increased by Adjuvant Alternating Electric Fields (TTFields), BMC Med. Phys., 9,I.

Lee, S.H., Choi, E.H., Feingold, K.R., Jiang, S. and Ahn, S.K., 1998, lontophoresis Itself on Hairless Mouse Skin Induces the Loss of the Epidermal Calcium Gradient Without Skin Barrier Impairment, J. Invest. Dermatol., I I I(I), 39-43.

Leslie, A., Carey, F.A., Pratt, N.R. and Steele, R.J.C., 2002, The Colorectal Adenoma-Carcinoma Sequence, Br. J. Surg., 89(7), 845-860.

Ma, W., Shi, T., Tang, Z., Liu, S., Malik, R. and Zhang, L., 20II, High-throughput Dielectrophoretic Manipulation of Bioparticles Within Fluids Through Biocompatible Three-Dimensional Microelectrode Array, Electrophoresis, 32(5), 494-505.

Mahaworasilpa, T.L., Coster, H.G.L. and George, E. P., 1996, Forces on Biological Cells Due to Applied 
Alternating (AC) Electric Fields, Biochim. Biophys. Acta., II 93(I), II8126.

Mursilatun, 2010,The Effect of External Electric Field ECCT on Breast Cancer Cell Proliferation (MVF-7), Thesis, Dept. of Physics University of Indonesia, Jakarta.

Pasquier, E. and Kavallaris, M., 2007, Microtubules: a Moving Target in Cancer Therapy, IUBMB IIFE, 60(3), 165-70.

Qiao, G., Duan, W., Chatwin, C., Sinclair, A. and Wang, W., 2010, Electrical Properties of Breast Cancer Cells from Impedance Measurement of Cell Suspensions, J. Phys. Conf. Ser., 224(I), 012081.

Sabrina, Q., 2014, Effect of Electric Field Treatment Variation Against Lethal Level Cell Line MCF-7 (human breast cancer) in Vitro and Measurement of Cell Capacitance Value, Thesis, Dept. of Physics University of Indonesia, Jakarta. Shinomiya, N., 200I, New Concepts in Radiation-Induced Apoptosis: 'Premitotic Apoptosis' and 'Postmitotic Apoptosis', J. Cell. Mol. Med., 5(3), 240253.
Takashima, S. and Schwan, H.P., 1985, Alignment of Microscopic Particles in Electric Fields and Its Biological Implications, Biophys. J., 47, 5I3-5I8.

Teo, Z.Y.P., 2003, The Role of Macrophages in Apoptosis; Initiator, Regulator, Scavenger, Reviews in Undergraduate Res., 2, 7-II.

Tsutsui, H. and Ho, C.M., 2009, Cell Separation by Non-inertial Force Fields in Microfluidic Systems, Mech. Res. Commun., 36(I), 92-103.

Weigert, A., Tzieply, N., Von Knethen, A., Johann, A. M., Schmidt, H., Geisslinger, G., et al., 2007, Tumor Cell Apoptosis Polarizes Macrophages-Role of Sphingosine-I-phosphate, Mol. Biol. Cell, 18(10), 3810-3819.

Zhao, M., Forrester, J.V. and McCaig, C.D., 1999, A Small, Physiological Electric Field Orients Cell Division, Proc. Natl. Acad. Sci. USA, 96(9), 4942-4946.

Zimmermann, U., Vienken, J. and Pilwat, G., 1981, Rotation of Cells in Alternating Electric Field: The Occurrence of a Resonance Frequency, Z. Naturforsch C., 36(I-2), I73-I77. 